PROCEEDINGS OF THE

AMERICAN MATHEMATICAL SOCIETY

Volume 133, Number 6, Pages 1733-1740

S 0002-9939(04)07741-X

Article electronically published on November 19, 2004

\title{
TRANSFERENCE FOR AMENABLE ACTIONS
}

\author{
WALDEMAR HEBISCH AND M. GABRIELLA KUHN \\ (Communicated by Andreas Seeger)
}

\begin{abstract}
Suppose $G$ acts amenably on a measure space $X$ with quasiinvariant $\sigma$-finite measure $m$. Let $\sigma$ be an isometric representation of $G$ on $L^{p}(X, d m)$ and $\mu$ a finite Radon measure on $G$. We show that the operator $\sigma_{\mu} f(x)=\int_{G}(\sigma(g) f)(x) d \mu(g)$ has $L^{p}(X, d m)$-operator norm not exceeding the $L^{p}(G)$-operator norm of the convolution operator defined by $\mu$. We shall also prove an analogous result for the maximal function $M$ associated to a countable family of Radon measures $\mu_{n}$.
\end{abstract}

\section{INTRODUCTION}

Let $\sigma$ be a uniformly bounded representation of a locally compact group $G$ on some Banach space $B$ and $K$ a convolution operator on $L^{p}(G)$ given by

$$
K f(x)=\int_{G} k(y) f\left(y^{-1} x\right) d y=k * f(x)
$$

with $k \in L^{1}(G)$. The transferred operator $K^{\sharp}$ is defined by letting

$$
K^{\sharp}(F)=\int_{G} k(y)(\sigma(y) F) d y .
$$

The basic transference theory goes back to the earliest works of R. Coifman and G. Weiss around 1970 and says that if we have an action of an amenable group $G$ on some ( $\sigma$-finite) measure space $X$ and we let $B=L^{p}(X)$, then

$$
\left\|K^{\sharp}\right\|_{L^{p}(X) \rightarrow L^{p}(X)} \leq\|K\|_{L^{p}(G) \rightarrow L^{p}(G)} .
$$

This theory has many different applications (it allows us to convert $L^{p}(\mathbb{T})$-Fourier multipliers into $L^{p}(\mathbb{R})$-Fourier multipliers, to bound the Riesz transform on $S U(2)$ or, more generally, on compact Lie groups having a "nice" maximal torus) and has been generalized to different contexts: the central role is played by an amenable group.

In $1978 \mathrm{R}$. Zimmer [8] introduced the notion of amenable action for locally compact topological groups $G$. When $X$ is a homogeneous $G$ space, say $X=G / H$, amenability of the action is equivalent to the amenability of $H$.

In this paper we shall prove that the above inequality (1) is true when $G$ acts amenably on a measure space $X$ with quasi-invariant $\sigma$-finite measure. We shall also

Received by the editors July 19, 2003 and, in revised form, February 15, 2004.

2000 Mathematics Subject Classification. Primary 47A30; Secondary 37A15, 43A07.

The first author was supported by KBN: 5 P03A 05020 and RTN: HPRN-CT-2001-00273, and partially by GNAMPA.

(C)2004 American Mathematical Society Reverts to public domain 28 years from publication 
prove an analogous result for the maximal function $M_{\sigma}$ with respect to a countable family of Radon measures $\mu_{n}$ on $G$.

\section{Notation AND RESUlts}

Let $G$ be a second countable locally compact group acting on a Borel space $X$ with quasi-invariant $\sigma$-finite measure $m$. We shall assume that $G$ is acting on the right, that is, $(g, x) \in G \times X \rightarrow g^{-1} x$. Let $r(g, x)$ be the Radon-Nikodym derivative of the $G$ action:

$$
r(g, x)=\frac{d m_{g}(x)}{d m} \quad \text { where } m_{g}(E)=m\left(g^{-1} E\right) ;
$$

equivalently,

$$
\int_{X}\left|f\left(g^{-1} x\right)\right| r(g, x) d m(x)=\int_{X}|f(x)| d m(x)
$$

for every measurable function $f$. Recall that $r$ can be chosen to be a strict Borel cocycle, that is, $r(g s, x)=r(g, x) r\left(s, g^{-1} x\right)$ for all $g, s \in G$ and $x \in X$.

We shall assume that $m$ is a probability measure, so that

$$
\int_{X} r(g, x) d m(x)=1 \quad \text { for all } g \in G \text {. }
$$

This can be done by choosing appropriately a representative in the measure class of $m$.

Let $\alpha$ be a cocycle, that is, a Borel function from $G \times X \rightarrow \mathbf{C}$ such that:

$$
\alpha(g s, x)=\alpha(g, x) \alpha\left(s, g^{-1} x\right) .
$$

In the sequel we will assume that $\alpha$ is unitary, that is, $|\alpha(g, x)|=1$.

For any given $g \in G$ define a norm preserving map $\sigma(g): L^{p}(X) \rightarrow L^{p}(X)$ by letting

$$
\sigma(g) f(x)=f\left(g^{-1} x\right)(r(g, x))^{\frac{1}{p}} \alpha(g, x) .
$$

Denote by $L^{p}(G \times X)$ the usual $L^{p}$ space with respect to the product measure $d g \times d m$ where $d g$ is the left invariant Haar measure on $G$.

Denote by $\lambda$ the left regular representation of $G$ acting on $L^{p}(G)$. Define a representation $\lambda \otimes \sigma$ on $L^{p}(G \times X)$ by the rule

$$
(\lambda \otimes \sigma)(g) F(h, x)=F\left(g^{-1} h, g^{-1} x\right)(r(g, x))^{\frac{1}{p}} \alpha(g, x) .
$$

Choose any finite Radon measure $\mu$ on $G$. Define operators $(\lambda \otimes \sigma)_{\mu}, \lambda_{\mu}$ and $\sigma_{\mu}$ acting respectively on $L^{p}(G \times X), L^{p}(G)$ and $L^{p}(X)$ according to the following rules:

$$
\begin{gathered}
(\lambda \otimes \sigma)_{\mu} F(h, x)=\int_{G} \lambda \otimes \sigma(g) F(h, x) d \mu(g) \int_{G} F\left(g^{-1} h, g^{-1} x\right)(r(g, x))^{\frac{1}{p}} \alpha(g, x) d \mu(g), \\
\lambda_{\mu} f(h)=\int_{G} \lambda(g) f(h) d \mu(g)=\int_{G} f\left(g^{-1} h\right) d \mu(g), \\
\sigma_{\mu} f(x)=\int_{G} \sigma(g) f(x) d \mu(g)=\int_{G} f\left(g^{-1} x\right)(r(g, x))^{\frac{1}{p}} \alpha(g, x) d \mu(g) .
\end{gathered}
$$

We shall denote by $\|\cdot\|_{p}$ the $L^{p}$ norm in any of the above-defined $L^{p}$ spaces and by $\|\cdot\|_{p, p}$ the norm of any of the above-defined operators.

When $G$ is amenable it is known (see e.g. [2], [3]) that $\left\|\sigma_{\mu}\right\|_{p, p} \leq\left\|\lambda_{\mu}\right\|_{p, p}$. This phenomenon is called transference. 
When $p=2$ and $G$ acts amenably on $X$ the same inequalities are true: see [1], Theorem 3.2.3 (for discrete groups see also [5] for ergodic actions and [6] for general amenable actions including semisimple algebraic groups).

In this paper we shall prove transference for all $p(1 \leq p<\infty)$.

We refer to Zimmer's book [9] for the general definition of amenable action.

For the purposes of this paper we need the following property of amenable $G$ spaces:

Lemma 2.1 (C. Anantharaman-Delaroche, 1], Theorem 3.1.5). Assume that $G$ is acting amenably on a standard Borel space with quasi-invariant measure $m$.

Then there exists a sequence $\phi_{i}$ of nonnegative functions $\phi_{i}(g, x)$ in $L^{1}(G \times X)$ such that for all $f \in L^{1}(X)$ and any compact subset $K \subset G$ we have

a) $\int_{G} \phi_{i}(g, x) d g=1$ for almost all $x \in X$,

b) $\lim _{i}\left(\sup _{k \in K} \int_{G} \int_{X}\left|\phi_{i}\left(k^{-1} g, k^{-1} x\right)-\phi_{i}(g, x)\right||f(x)| d m(x) d g\right)=0$.

Remark 2.2. When $G$ is discrete and ergodic on $X$, the existence of such a sequence was essentially the proof (given in [5]) that the quasi-regular representation of $G$ on $X$ is weakly contained into the regular representation of $G$. In [1] it is proved that the above condition is in fact equivalent to the amenabilty of the $G$ action on $X$ for any locally compact second-countable group.

Fix once and for all a sequence $\phi_{i}$ of nonnegative functions satisfying $\mathbf{a}$ ) and $\mathbf{b}$ ) of Lemma 2.1.

For every $f \in L^{p}(X)$ and $i \in \mathbb{N}$ define a function $\tilde{f}_{i} \in L^{p}(G \times X)$ according to the following rule:

$$
\tilde{f}_{i}(g, x)=\left(\phi_{i}(g, x)\right)^{\frac{1}{p}} f(x)
$$

so that

$$
\widetilde{\sigma_{\mu} f}(g, x)=\left(\phi_{i}(g, x)\right)^{\frac{1}{p}}\left(\sigma_{\mu} f\right)(x) .
$$

Lemma 2.3. Let $\mu$ be any finite Radon measure and $f \in L^{p}(X)$. Let $\tilde{f}_{i}$ and $\widetilde{\sigma_{\mu} f}{ }_{i}$ be as above.

Then we have

$$
\lim _{i}\left\|(\lambda \otimes \sigma)_{\mu} \tilde{f}_{i}-{\widetilde{\sigma_{\mu}}}_{i}\right\|_{p}=0 .
$$

Proof. First, we will assume that $\mu$ has compact support $F$. Choose $\varepsilon>0$ and fix $f$. By Lemma 2.1 (with $K=F^{-1}$ ) we can find $N$ such that for all $i \geq N$,

$$
\begin{gathered}
\int_{G} \phi_{i}(g, x) d g=1, \\
\sup _{k \in F}\left(\int_{G} \int_{X}\left|\phi_{i}(k g, k x)-\phi_{i}(g, x)\right||f(x)|^{p} d m(x) d g\right)<\varepsilon .
\end{gathered}
$$

We need to compute the norm in $L^{p}(G \times X)$ of the quantity

$$
\begin{aligned}
\left((\lambda \otimes \sigma)_{\mu} \tilde{f}_{i}-\widetilde{\sigma_{\mu} f}\right. & )(g, x) \\
& =\int_{F}\left\{\left(\phi_{i}\left(k^{-1} g, k^{-1} x\right)\right)^{\frac{1}{p}}-\left(\phi_{i}(g, x)\right)^{\frac{1}{p}}\right\}\{r(k, x)\}^{\frac{1}{p}} \alpha(k, x) f\left(k^{-1} x\right) d \mu(k) .
\end{aligned}
$$


Denote by $|\mu|$ the measure total variation of $\mu$. Using Minkowski's integral inequality we get

$$
\begin{gathered}
\left\{\int_{G \times X}\left|(\lambda \otimes \sigma)_{\mu} \tilde{f}_{i}-{\widetilde{\sigma_{\mu}}}_{i}\right|^{p}(g, x) d(g) d m(x)\right\}^{\frac{1}{p}} \\
\leq \int_{F} d|\mu|(k)\left\{\int_{G \times X} \mid\left(\phi_{i}\left(k^{-1} g, k^{-1} x\right)\right)^{\frac{1}{p}}\right. \\
=\int_{F}\left\{\int_{G \times X}\left|\phi_{i}(k g, k x)^{\frac{1}{p}}-\phi_{i}(g, x)^{\frac{1}{p}}\right|^{p}|f(x)|^{p} d(g) d m(x)\right\}^{\frac{1}{p}} d|\mu|(k) \\
\leq \int_{F}\left\{\int_{G \times X}\left|\phi_{i}(k g, k x)-\phi_{i}(g, x)\right||f(x)|^{p} d(g) d m(x)\right\}^{\frac{1}{p}} d|\mu|(k) \\
\leq \varepsilon^{\frac{1}{p}}\|\mu\|
\end{gathered}
$$

where for the last inequality (3) we used $\left|a^{\frac{1}{p}}-b^{\frac{1}{p}}\right|^{p} \leq|a-b|$ (valid for positive $a$ and $b)$ and $\|\mu\|$ stays for $|\mu|(G)$.

So, we showed that for fixed compactly supported $\mu$ and given $f$ we can choose $N$ such that for all $i \geq N$,

$$
\left\|(\lambda \otimes \sigma)_{\mu} \tilde{f}_{i}-\widetilde{\sigma_{\mu} f}\right\|_{i} \leq \varepsilon^{\frac{1}{p}}\|\mu\|,
$$

which gives our claim for compactly supported $\mu$.

Now, let $K_{n}$ be an increasing sequence of compact sets such that $G=\bigcup_{n} K_{n}$. Let $\mu_{n}$ denote the restriction of $\mu$ to $K_{n}$.

$$
\left\|\mu-\mu_{n}\right\|=\int_{K_{n}^{c}} d|\mu| \rightarrow 0
$$

because $|\mu|$ is finite and countably additive. For fixed $n$,

$$
\begin{aligned}
& \lim \sup _{i}\left\|(\lambda \otimes \sigma)_{\mu} \tilde{f}_{i}-\widetilde{\sigma_{\mu} f_{i}}\right\|_{p} \\
& \leq \lim \sup _{i}\left(\left\|(\lambda \otimes \sigma)_{\mu_{n}} \tilde{f}_{i}-\widetilde{\sigma_{\mu_{n}} f_{i}}\right\|_{p}+\|f\|_{p}\left\|\mu-\mu_{n}\right\|\right) \\
& =\|f\|_{p}\left\|\mu-\mu_{n}\right\| \text {. }
\end{aligned}
$$

Since $\left\|\mu-\mu_{n}\right\|$ is arbitrarly small when $n$ is large we get the claim.

We are now able to prove our result:

Theorem 2.4. Let $G$ be a second-countable locally compact group acting amenably on a standard Borel space with quasi-invariant $\sigma$-finite measure $m$. Fix a finite Radon measure $\mu$ on $G$. Define, as before,

$$
\sigma_{\mu} f(x)=\int_{G} f\left(g^{-1} x\right)(r(g, x))^{\frac{1}{p}} \alpha(g, x) d \mu(g) .
$$

Then the operator norm of $\sigma_{\mu}$ acting on $L^{p}(X)$ is bounded by the operator norm of $\lambda_{\mu}$ acting on $L^{p}(G)$. In other words,

$$
\left\|\sigma_{\mu}\right\|_{p, p} \leq\left\|\lambda_{\mu}\right\|_{p, p}
$$

Proof. Define

$$
(U F)(g, x)=F(g, g x)\left(r\left(g^{-1}, x\right)\right)^{\frac{1}{p}} \alpha\left(g^{-1}, x\right)=\sigma\left(g^{-1}\right) F(g, x)
$$


for every $F \in L^{p}(G \times X)$. Observe that $U$ intertwines $\lambda \otimes \sigma$ to $\lambda \otimes \mathrm{i}$ where

$$
(\lambda \otimes \mathrm{i})(g) F(h, x)=F\left(g^{-1} h, x\right) .
$$

Observe that $U^{-1}$ is also well defined:

$$
U^{-1} F(g, x)=\sigma(g) F(g, x)
$$

and $\|U F\|_{p}=\|F\|_{p}=\left\|U^{-1} F\right\|_{p}$, so that $\left\|(\lambda \otimes \sigma)_{\mu}\right\|_{p, p}=\left\|(\lambda \otimes \mathrm{i})_{\mu}\right\|_{p, p}$. Since obviously $\left\|(\lambda \otimes \mathrm{i})_{\mu}\right\|_{p, p} \leq\left\|\lambda_{\mu}\right\|_{p, p}$, it is enough to compare the norm of $\sigma_{\mu}$ with the norm of $(\lambda \otimes \sigma)_{\mu}$.

Fix $f \in L^{p}(X)$. Let $\tilde{f}_{i}$ and $\widetilde{\sigma_{\mu} f}$ be as in Lemma 2.3. Observe that

$$
\|f\|_{p}=\left\|\tilde{f}_{i}\right\|_{p} \quad \text { and } \quad\left\|\sigma_{\mu} f\right\|_{p}=\left\|\widetilde{\sigma_{\mu} f}\right\|_{p} .
$$

Since

$$
\begin{aligned}
& \left\|\widetilde{\sigma_{\mu} f_{i}}\right\|_{p} \leq\left\|(\lambda \otimes \sigma)_{\mu} \tilde{f}_{i}\right\|_{p}+\left\|(\lambda \otimes \sigma)_{\mu} \tilde{f}_{i}-\widetilde{\sigma_{\mu} f_{i}}\right\|_{p} \\
& \leq\left\|(\lambda \otimes \sigma)_{\mu}\right\|_{p, p}\left\|\tilde{f}_{i}\right\|_{p}+\left\|(\lambda \otimes \sigma)_{\mu} \tilde{f}_{i}-\widetilde{\sigma_{\mu} f_{i}}\right\|_{p} \\
& =\left\|(\lambda \otimes \sigma)_{\mu}\right\|_{p, p}\|f\|_{p}+\left\|(\lambda \otimes \sigma)_{\mu} \tilde{f}_{i}-\widetilde{\sigma_{\mu} f_{i}}\right\|_{p},
\end{aligned}
$$

we also have

$$
\left\|\sigma_{\mu} f\right\|_{p} \leq\left\|(\lambda \otimes \sigma)_{\mu}\right\|_{p, p}\|f\|_{p}+\left\|(\lambda \otimes \sigma)_{\mu} \tilde{f}_{i}-\widetilde{\sigma_{\mu} f}\right\|_{p} .
$$

Taking the limit as $i \rightarrow \infty$ we get

$$
\left\|\sigma_{\mu} f\right\|_{p} \leq\left\|(\lambda \otimes \sigma)_{\mu}\right\|_{p, p}\|f\|_{p}
$$

which gives

$$
\left\|\sigma_{\mu} f\right\|_{p} \leq\left\|(\lambda \otimes \sigma)_{\mu}\right\|_{p, p} \quad\|f\|_{p}=\left\|(\lambda \otimes \mathrm{i})_{\mu}\right\|_{p, p} \quad\|f\|_{p} \leq\left\|\lambda_{\mu}\right\|_{p, p} \quad\|f\|_{p} .
$$

Remark 2.5. As an application take $G=S L(2, R)$ and $X=P^{1}$, the projective line. Since $X \simeq S L(2, R) / P$ where $P=\left(\begin{array}{ll}a & 0 \\ b & a^{-1}\end{array}\right), X$ is an amenable $G$ space. The $G$ action is given by fractional linear transformations

$$
g^{-1} x=\frac{a x+b}{c x+d} \quad \text { for } g^{-1}=\left(\begin{array}{ll}
a & b \\
c & d
\end{array}\right),
$$

and the Radon-Nikodým derivative $r(g, x)$ is given by $r(g, x)=\frac{1}{(c x+d)^{2}}$. For every $f \in L^{p}(X)$ define

or, more generally,

$$
R_{t} f(x)=\frac{f\left(g^{-1} x\right)}{(c x+d)^{\frac{2}{p}+i t}}
$$

$$
R_{\mu} f(x)=\int_{G} \frac{f\left(g^{-1} x\right)}{(c x+d)^{\frac{2}{p}+i t}} d \mu(g) .
$$

Then $R_{t}$ is an isometry for every real $t$ and $1 \leq p<\infty$, while $\left\|R_{\mu}\right\| \leq\left\|\lambda_{\mu}\right\|$.

More generally, we can apply our result to boundaries of symmetric spaces: $X=G / M A N$ (so we can transfer to the corresponding principal series representations).

We can also handle symmetric spaces: $X=G / K$ (however, since $K$ is compact, a more direct argument works better). 
Remark 2.6. Assume that $\mu$ (not necessarily given by a Radon measure) is a bounded convolver of $L^{p}(G)$ for some $p(1 \leq p<\infty)$. Suppose also that $G$ is amenable: another phenomenon related to transference says that such a $\mu$ is also a bounded convolver of $L^{2}(G)$ (see [4]). We want to remark that such a phenomenon does not hold in the case of amenable actions: take the free group $F_{r}$ on $r$ generators $(r \geq 2)$ acting on itself; this action is amenable since the subgroup $\{e\}$ is compact and $X=F_{r} / e$. It is well known that there exists a bounded convolver of $\ell^{p}\left(F_{r}\right)$ that is not a convolver of $\ell^{q}\left(F_{r}\right)$ for any $q \neq p$; see [7. Moreover, in the same paper, it is shown that for every positive $M$ there exists a function $f$ with finite support such that $\left\|\lambda_{f}\right\|_{2,2}>M\left\|\lambda_{f}\right\|_{p, p}$ ( $\lambda$ denotes, as usual, the left regular representation of $F_{r}$ ).

Theorem 2.7. Let $G$ and $X$ be as in Theorem 2.4. Fix any sequence $\left\{\mu_{n}\right\}$ of finite Radon measures on $G$. Define

$$
M_{\sigma} f(x)=\sup _{n}\left|\sigma_{\mu_{n}} f\right|(x)=\sup _{n}\left|\int_{G} f\left(g^{-1} x\right)(r(g, x))^{\frac{1}{p}} \alpha(g, x) d \mu_{n}(g)\right| .
$$

Analogously define

$$
M_{\lambda} f(h)=\sup _{n}\left|\lambda_{\mu_{n}} f\right|(h)=\sup _{n}\left|\int_{G} f\left(g^{-1} h\right) d \mu_{n}(g)\right| .
$$

Then the operator norm of $M_{\sigma}$ acting on $L^{p}(X)$ is bounded by the operator norm of the corresponding $M_{\lambda}$ acting on $L^{p}(G)$.

Sketch of the proof. Notation as in Theorem 2.4. It is enough to show that, for every positive integer $L$,

$$
\left\|M_{\{\sigma, L\}} f\right\|_{p} \leq\left\|M_{\{\lambda, L\}}\right\|_{p, p}\|f\|_{p}
$$

where

$$
M_{\{\sigma, L\}} f(x)=\sup _{1 \leq n \leq L}\left|\sigma_{\mu_{n}} f\right|(x)
$$

and $M_{\{\lambda, L\}}$ is defined analogously. Let

$$
\begin{gathered}
\widetilde{M_{\{\sigma, L\}}} f_{i}(g, x)=\left(\phi_{i}(g, x)\right)^{\frac{1}{p}} M_{\{\sigma, L\}} f(x) \\
=\left(\phi_{i}(g, x)\right)^{\frac{1}{p}} \sup _{1 \leq n \leq L}\left|\int_{G} f\left(g^{-1} x\right)(r(g, x))^{\frac{1}{p}} \alpha(g, x) d \mu_{n}(g)\right| \\
=\sup _{1 \leq n \leq L}\left|\left(\phi_{i}(g, x)\right)^{\frac{1}{p}} \int_{G} f\left(g^{-1} x\right)(r(g, x))^{\frac{1}{p}} \alpha(g, x) d \mu_{n}(g)\right| \\
=\sup _{1 \leq n \leq L}\left|\widetilde{\sigma_{\mu_{n}} f_{i}}\right|(x) .
\end{gathered}
$$

Again we have

$$
\left\|M_{\{\sigma, L\}} f\right\|_{p}=\left\|\widetilde{M_{\{\sigma, L\}} f}\right\|_{i} .
$$

Remember the definition of $U^{-1}: L^{p}(G \times X) \rightarrow L^{p}(G \times X)$ :

$$
\left(U^{-1}\right) F(g, x)=F\left(g, g^{-1} x\right)(r(g, x))^{\frac{1}{p}} \alpha(g, x)=\sigma(g) F(g, x) .
$$

Put

$$
V F(g, x)=F(g, g x)\left(r\left(g^{-1}, x\right)\right)^{\frac{1}{p}}
$$


so that

$$
\left(V^{-1}\right) F(g, x)=F\left(g, g^{-1} x\right)(r(g, x))^{\frac{1}{p}}
$$

and $V$ is an isometry.

Observe that

$$
V^{-1}\left(\sup _{n}\left|F_{n}\right|\right)=\sup _{n}\left(\left|U^{-1} F_{n}\right|\right) .
$$

For every $f \in L^{p}(G \times X)$ write $f=U^{-1} F$ and evaluate

$$
\begin{array}{r}
M_{\{\lambda \otimes \sigma, L\}} f(g, x)=\sup _{1 \leq n \leq L}\left|(\lambda \otimes \sigma)_{\mu_{n}} f\right|(g, x)=\sup _{1 \leq n \leq L}\left|(\lambda \otimes \sigma)_{\mu_{n}} U^{-1} F\right|(g, x) \\
=\sup _{1 \leq n \leq L}\left|U^{-1}(\lambda \otimes \mathrm{i})_{\mu_{n}} F\right|(g, x)=V^{-1} \sup _{1 \leq n \leq L}\left|(\lambda \otimes \mathrm{i})_{\mu_{n}} F\right|(g, x) \\
=V^{-1}\left(M_{\{\lambda \otimes \mathrm{i}, L\}}\right) F(g, x) .
\end{array}
$$

Hence

$$
\left\|M_{\{\lambda \otimes \sigma, L\}}\right\|_{p, p}=\left\|M_{\{\lambda \otimes \mathrm{i}, L\}}\right\|_{p, p} \leq\left\|M_{\{\lambda, L\}}\right\|_{p, p} .
$$

Compute

$$
\begin{aligned}
\left\|M_{\{\sigma, L\}} f\right\|_{p}=\left\|\widetilde{M_{\{\sigma, L\}}} f_{i}\right\|_{p} \\
\quad \leq\left\|\widetilde{M_{\{\sigma, L\}}} f_{i}-M_{\{\lambda \otimes \sigma, L\}} \tilde{f}_{i}\right\|_{p}+\left\|M_{\{\lambda \otimes \sigma, L\}}\right\|_{p, p}\|f\|_{p} .
\end{aligned}
$$

Since

(7)

$$
\left|\sup _{1 \leq n \leq L}\right| \widetilde{\sigma_{\mu_{n}} f_{i}}\left|(g, x)-\sup _{1 \leq n \leq L}\right|(\lambda \otimes \sigma)_{\mu_{n}} \tilde{f}_{i}|(g, x)| \leq \sum_{n=1}^{L} \widetilde{\mid \sigma_{\mu_{n}} f_{i}}-(\lambda \otimes \sigma)_{\mu_{n}} \tilde{f}_{i} \mid(g, x),
$$

one has

$$
\left\|\widetilde{M_{\{\sigma, L\}}} f_{i}-M_{\{\lambda \otimes \sigma, L\}} \tilde{f}_{i}\right\|_{p} \leq \sum_{n=1}^{L}\left\|\widetilde{\sigma_{\mu_{n}} f}-(\lambda \otimes \sigma)_{\mu_{n}} \tilde{f}_{i}\right\|_{p} \rightarrow 0 .
$$

Conclude the proof as in Theorem 2.4.

\section{ADDED AFTER PROOF}

After this paper was accepted the authors were informed by A. Nevo that there was an old preprint of S. Durand and R. Feres which, using different language, presents very similar ideas.

\section{REFERENCES}

[1] C. Anantharaman-Delaroche ; On spectral characterization of amenability, Israel J. Math. 137, (2003), 1-33. MR2013348

[2] R. Coifman, G. Weiss ; Operators associated with representations of amenable groups singular integrals induced by ergodic flows, the rotation methods and multipliers. Studia Math. 47, (1973), 285-303. MR0336233 (49:1009)

[3] R. Coifman, G. Weiss ; Transference methods in analysis. C.B.M.S. Regional Conference Series in Math. 31, AMS Providence 1-59 (1977) reprinted in 1986. MR0481928 (58:2019)

[4] C. Herz; The theory of $p$-spaces with an application to convolution operators. Trans. Amer. Math. Soc. 154, (1971), 69-82. MR0272952 (42:7833)

[5] M.G. Kuhn ; Amenable actions and weak containment of certain representations of discrete groups. Proc. Amer. Math. Soc. 122, (1994) 751-757. MR1209424 (95a:43002)

[6] A. Nevo ; The spectral theory of amenable actions and invariants of discrete groups, Geom. Dedicata 100, (2003), 187-218. MR.2011122 
[7] T. Pytlik ; A construction of convolution operators on free group. Studia Math. 74, (1984), 73-76. MF 0772006 (86e:43010)

[8] R. Zimmer ; Amenable ergodic group actions and an application to Poisson boundaries of random walks. J. Func. Anal. 27 (1978), 350-372. MR0473096 (57:12775)

[9] R. Zimmer ; Ergodic theory and semisimple groups. Birkhäuser Boston 1984. MR0776417 (86j:22014)

Mathematical Institute, Wroceaw University, Pl. Grunwaldzki 2/4, 50-384 WrocŁaW, POLAND

E-mail address: hebisch@math.uni.wroc.pl

Dipartimento di Matematica, Università di Milano "Bicocca", Via R. Cozzi 53, EdifiCio U5, 20125 Milano, Italia

E-mail address: kuhn@matapp.unimib.it 